\title{
Erratum
}

\section{Les caractéristiques épidémiologiques et cliniques des envenimations scorpioniques dans la province de Beni Mellal (Maroc)}

\section{Ann Toxicol Anal. 2009; 21(3): 143-146}

Nezha Charrab, Abdelmajid Soulaymani ${ }^{\star}$, lham Semlali, Abdelghani Mokhtari, Rhizlane El Oufir, Rachida Soulaymani Bencheikh

Reçu le 4 janvier 2010

Les accidents provoqués par les piqûres de scorpions sont fréquents dans certains pays, en particulier, en Amérique Centrale, en Amérique du Sud, en Afrique du Nord, au Moyen-Orient et en Inde [1,2]. Au Maroc, les piqûres scorpioniques sont la première cause des intoxications déclarées au Centre Antipoison et de Pharmacovigilance du Maroc (CAPM). Cette pathologie occupe $5 \%$ de l'activité des Urgences et 6,3\% de l'activité des services de réanimation avec un taux d'occupation hospitalière spécifique de 3,5\% [3]. La province de Beni Mellal est une zone à forte incidence, connue par sa richesse en espèces scorpioniques et parmi ces espèces, celles de la famille des buthidés sont les plus incriminées, en particulier Androctonus mauretanicus, Buthus occitanus et Hottentota franzwerneri, à l'exception de l'espèce Scorpio maurus qui appartient à la famille des scorpionidés. L'objectif de notre travail est d'analyser les données des fiches d'hospitalisation et de déterminer les facteurs de risques afin de diminuer la morbidité et la mortalité et d'améliorer la prise en charge des patients envenimés.

\section{Références}

1. Krifi N, Kharrat H, El ayab M. Evaluation of antivenom therapy in children severely envenomed by Androctonus australis garzonii (Aag) and Buthus occitanus tunetanus (Bot) scorpions. Toxicon. 1999; 37: 1627-1634.

2. Devaux C, Jouiron B, Naceur Krifi M, Clot-Faybesse O, El Ayeb M, Rochat H. Quantitative variability in the biodistribution and in toxinokinetic studies of the three main alpha toxins from the androctonus australis hectors scorpion venom: Toxicon. 2004; 43: 661-669.

3. Charrab N, Soulaymani A, Mokhtari A, Soulaymani R. Les envenimations scorpioniques à l'hôpital provincial de Beni Mellal (Maroc). Med Trop. 2009; 69: 33-36.

\footnotetext{
^Correspondance : Abdelmajid Soulaymani, soulaymani@ hotmail.com
} 